\title{
Type 2 Autoimmune Lymphoproliferative Syndrome
}

National Cancer Institute

\section{Source}

National Cancer Institute. Type 2 Autoimmune Lymphoproliferative Syndrome. NCI

Thesaurus. Code C39576.

A rare, primary immunodeficiency with an autosomal dominant pattern of inheritance but incomplete penetrance. It is caused by a mutation in the CASP10 (caspase-10) gene that leads to defective Fas-induced apoptosis. Disruption of Fas-induced apoptosis impairs lymphocyte homeostasis and immune tolerance. Characteristic laboratory findings include an increase in circulating, double-negative (CD4-/CD8-) T cells in the setting of immune-mediated anemia, thrombocytopenia and neutropenia. Clinical signs present in childhood include fatigue, pallor, bruising, hepatosplenomeg aly and chronic, nonmalignant, non-infectious lymphadenopathy. The clinical course is influenced by a strong association with other autoimmune disorders and an increased risk for developing Hodgkin and non-Hodgkin lymphoma. 